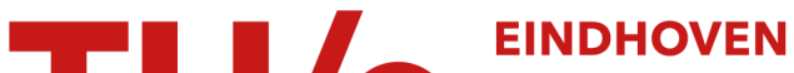

\section{An experimental digital consumer HDTV recorder using MC- DCT video compression}

Citation for published version (APA):

With, de, P. H. N., Rijckaert, A. M. A., Keesen, H-W., Kaaden, J., \& Opelt, C. (1993). An experimental digital consumer HDTV recorder using MC-DCT video compression. IEEE Transactions on Consumer Electronics, 39(4), 711-722. https://doi.org/10.1109/30.267390

DOI:

$10.1109 / 30.267390$

Document status and date:

Published: 01/01/1993

\section{Document Version:}

Publisher's PDF, also known as Version of Record (includes final page, issue and volume numbers)

\section{Please check the document version of this publication:}

- A submitted manuscript is the version of the article upon submission and before peer-review. There can be important differences between the submitted version and the official published version of record. People interested in the research are advised to contact the author for the final version of the publication, or visit the $\mathrm{DOI}$ to the publisher's website.

- The final author version and the galley proof are versions of the publication after peer review.

- The final published version features the final layout of the paper including the volume, issue and page numbers.

Link to publication

\section{General rights}

Copyright and moral rights for the publications made accessible in the public portal are retained by the authors and/or other copyright owners and it is a condition of accessing publications that users recognise and abide by the legal requirements associated with these rights.

- Users may download and print one copy of any publication from the public portal for the purpose of private study or research.

- You may not further distribute the material or use it for any profit-making activity or commercial gain

- You may freely distribute the URL identifying the publication in the public portal.

If the publication is distributed under the terms of Article 25fa of the Dutch Copyright Act, indicated by the "Taverne" license above, please follow below link for the End User Agreement:

www.tue.nl/taverne

Take down policy

If you believe that this document breaches copyright please contact us at:

openaccess@tue.nl

providing details and we will investigate your claim. 


\section{AN EXPERIMENTAL DIGITAL CONSUMER HDTV RECORDER USING MC-DCT VIDEO COMPRESSION}

\author{
P. H. N. de With \\ Philips Research Labs. \\ Eindhoven, The Netherlands
}

\author{
A. M. A. Rijckaert, H.-W. Keesen, and J. Kaaden \\ Thomson Consumer Electronics \\ Hanover, Germany
}

\author{
C. Opelt \\ Grundig R\&D \\ Fürth, Germany
}

\begin{abstract}
The concept and real-time implementation of an experimental home-use digital HDTV recorder is presented. An advanced motion-compensated DCT bit-rate reduction system is used to realize high-quality images at 50 $\mathrm{Mbit} / \mathrm{s}$ only, thereby enabling small recording mechanics for portable applications and featuring a new high-efficiency channel code with embedded tracking information.
\end{abstract}

\section{Introduction}

In the past years, several experimental digital recording systems have been worked out for standard-definition (SDTV) signals [1] at ca. 20 $\mathrm{Mbit} / \mathrm{s}$ net video bit rate. In this paper ${ }^{1}$ we explore the subsequent step, namely digital highdefinition video recording (HDTV) for consumer use, and we present an experimental HDTV recorder with a playing time of two hours at a net video bit rate of $50 \mathrm{Mbit} / \mathrm{s}$. The recorder concept, verified by a real-time prototype, is based on small magnetic recording mechanics and advanced bit-rate reduction techniques.

It needs no argument that digital recording is particularly suited for HDTV applications, since it offers intrinsically high time-base stability and flawless reproduction and copying of high-quality images. Bit-rate reduction is a key aspect of a consumer HDTV recorder, since the starting bit rate of HDTV signals, typically 400$500 \mathrm{Mbit} / \mathrm{s}$, is far too high to implement a cost-effective recording mechanism based on a

\footnotetext{
${ }^{1}$ The work reported in this paper was carried out in the framework of the European RACE 1001 DVT project and was sponsored by the European Community. The authors are responsible for the contents of the paper.
}

\begin{tabular}{|l|l|}
\hline \hline & $\mathrm{Y}, \mathrm{U}, \mathrm{V} / 1250,2: 1$ \\
video inputs & $54 \mathrm{MHz}$ \\
sampling U, V & $27 \mathrm{MHz}$ \\
compression unit & $2 \mathrm{frames}$ \\
frame 1 coding & intraframe DCT + VLC \\
frame 2 coding & motion compensation \\
HDTV bit rate & $50 \mathrm{Mbit} / \mathrm{s}$ \\
audio & 2 stereo channels \\
audio sampling & $48 \mathrm{kHz}, 16$ bits \\
error correction & $\mathrm{R}-\mathrm{S} \mathrm{product}$ code \\
channel code & $24-25 \mathrm{modulation}$ \\
recording rate & $72 \mathrm{Mbit} / \mathrm{s}$ \\
tracking & embedded tones \\
scanner & $2 \times 2 \mathrm{heads}$ \\
drum & $40 \mathrm{~mm}$ \\
track pitch & $10 \mu \mathrm{m}$ \\
tape & $8 \mathrm{~mm}, \mathrm{ME}$ \\
& \\
\hline \hline
\end{tabular}

Table 1. Key parameters of HDTV recorder.

small cassette.

Recently, experimental SDTV recorders have been reported using intraframe (or -field) Discrete Cosine Transform (DCT) coding for bitrate reduction. Borgers et al. [2] and Yoneda et al. [3] describe DCT coding of $8 \times 8$ sample blocks with feedback buffer control on 19 and $28 \mathrm{Mbit} / \mathrm{s}$, respectively. The difference in bit rate is explained by the applied sampling standards for YUV signals (3:1:1 versus 4:2:2). Doi et al. [4] and Yamamitsu et al. [5] reported on a system with feedforward bit-rate control yielding fixed rate on subimage basis, using 19-40 Mbit/s video bit rate. Finally, Onishi et al. [6] discussed a more complex system in which 4 consecutive frames of $4: 2: 2$ sampled images were compressed 


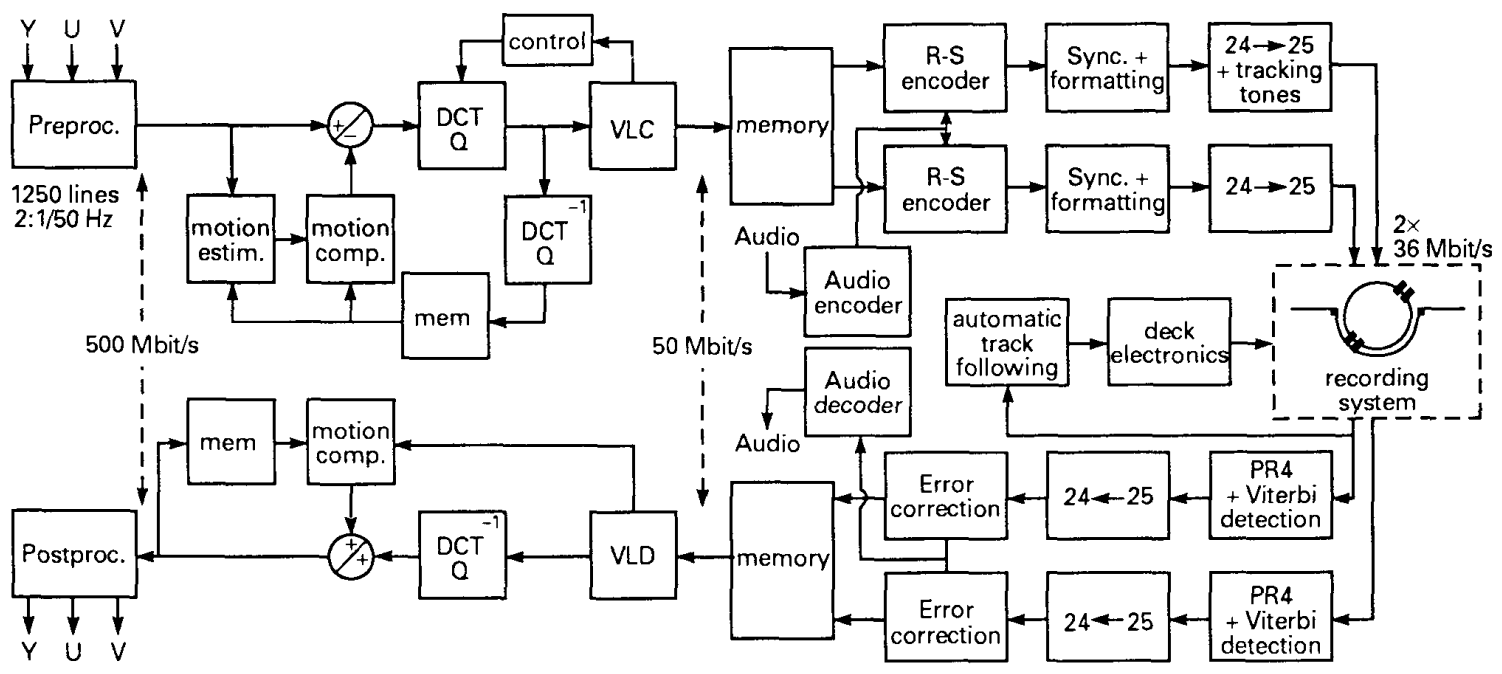

Figure 1: Block diagram of the experimental HDTV recorder.

to $23 \mathrm{Mbit} / \mathrm{s}$.

The aforementioned papers lead to the conclusion that SDTV recording on $25 \mathrm{Mbit} / \mathrm{s}$ is well realizable. In this paper, we extend the intraframe DCT technology to HDTV applications. However, in order to avoid a quadrapuled bit rate after compression for HDTV and the corresponding complex scanner, we have added motion-compensation techniques to obtain an extra compression factor of two, so that $50 \mathrm{Mbit} / \mathrm{s}$ net HDTV bit rate could be achieved.

From the historical development of recording systems, a clear trend towards smaller recording equipment can be observed. The recording mechanics are derived from the existing $8 \mathrm{~mm}$ system, which is considered small enough (e.g. also the cassette) to ensure portable applications. The scanner was designed to increase both the recording bit rate to $72 \mathrm{Mbit} / \mathrm{s}$ and the recording density by using special heads having a track width of $10 \mu \mathrm{m}$. Depending on the tape thickness, 1-2 hours playing time can be realized with the given system parameters. In contrast with our previous work [2], the bit rate is even more critical, so that a high-efficiency 24-25 channel code has been designed, which still offers digitally embedded tracking tones. Furthermore, substantial consideration was given to the feasibility of the system for the recording trick modes, such as editing and fast viewing and search.

\section{System architecture}

In this section we present the architecture of the recorder. The block diagram of the recorder is depicted in Figure 1. Table 1 portrays the key parameters of the recording system. The YUV video components are sampled at 54 and $27 \mathrm{MHz}$ with line-sequential processing for the colour-difference signals (16:8:0 sampling). The HDTV video signal is interlaced and one complete frame contains 1250 lines. For recording, the line- and field blanking are omitted, so that the input bit-rate is slightly less than $500 \mathrm{Mbit} / \mathrm{s}$.

The implications of a small cassette with sufficient playing time are that the recording bit rate is limited to $70-80 \mathrm{Mbit} / \mathrm{s}$, so that the HDTV signals must be compressed by a factor of 10 . As intraframe DCT coding yields a factor of 56 only, additional measures are required for further rate reduction. This has been achieved by compressing groups of two frames, rather than single frames, and by combining DCT coding with motion compensation. Independent coding of groups of two consecutive frames, henceforth referred to as intra-two-frame coding, allows for editing on two-frame basis. The bit-rate reduction is enhanced by motion-compensated coding of every second frame, while the first frame is compressed with the conventional intraframe DCT coding technique. Parts of this frame are then used for the fast viewing and search mode 
of the recorder.

The recording channel is based on a special scanner with $40 \mathrm{~mm}$ drum and $2 \times 2$ heads. In the drum, rotating preamplifiers have been mounted for improving the SNR. The scanner contains no actuator, although the tracking system allows for dynamic track following. The video data are recorded via two parallel channels using 36 Mbit/s recording bit rate for each channel (see Fig. 1, right-hand part). System robustness is improved substantially by using a Reed-Solomon product code for error correction. The code block consists of rows that are equal to sync blocks, while the number of rows is large enough to correct long burst errors. For further channel coding, we have implemented a new high-efficiency DC-free channel code, called 24-25 modulation, to constitute pilot tones for tracking which are embedded in the data.

\section{Bit-rate reduction}

\subsection{System constraints and sampling}

The choice for a particular coding algorithm needs to be adjusted to the specific system aspects of a VCR, such as editing, trick-play performance, error robustness and reliability. For the coding architecture, a trade-off should be made between the resulting picture quality and resolution and the hardware complexity. Moreover, the synergy with SDTV cannot be neglected, since this point will be very important in future, both for the consumer, desiring compatibility with existing (SDTV) software, and the manufacturer for reduction of production costs. For this purpose, and to enable the use of small cassettes, a net video bit rate of $50 \mathrm{Mbit} / \mathrm{s}$ for HDTV recordings (twice the rate of compressed SDTV) has been adopted. In this way, the channel electronics and scanner can be constructed with acceptable costs for consumer HDTV and an SDTV record-playback mode can be easily realized.

Prior to discussing the compression algorithm, the input sampling will be specified. Table 2 portrays possible sampling standards and indicates the compression ratio needed for a $50 \mathrm{Mbit} / \mathrm{s}$ output rate. Two sampling standards -marked with an 'S'- are particularly interesting, since they enable (theoretically) an HDTV tape format which is very similar to digital SDTV formats.

\begin{tabular}{|c|c|c|c|}
\hline $\begin{array}{c}\text { Sampling } \\
\text { frequency }\end{array}$ & $\begin{array}{c}\text { Sampling } \\
\text { standard }\end{array}$ & $\begin{array}{c}\text { Comp. } \\
\text { ratio }\end{array}$ & Remark \\
\hline $40.5 / 13.50$ & $12: 4: 0 \mathrm{~S}$ & 6.6 & $f_{s} \mathrm{Y} 3 \times \mathrm{SD}$ \\
$40.5 / 20.25$ & $12: 6: 0$ & 7.5 & $f_{s} \mathrm{Y} 3 \times \mathrm{SD}$ \\
$54.0 / 18.0$ & $16: 5.33: 0$ & 8.8 & $f_{s} \mathrm{Y} 4 \times \mathrm{SD}$ \\
$54.0 / 27.0$ & $16: 8: 0 \mathrm{~S}$ & 10.0 & $f_{s} \mathrm{Y} 4 \times \mathrm{SD}$ \\
\hline
\end{tabular}

Table 2. Possible sampling standards for consumer HDTV recording and corresponding compression ratios for $50 \mathrm{Mbit} / \mathrm{s}$ net video rate.

The compression ratios indicate that, even if the lowest HD sampling standard of 12:4:0 is adopted, the capabilities of straightforward intraframe coding will be exceeded. Provided that intraframe coding can hardly yield sufficient HDTV quality at $50 \mathrm{Mbit} / \mathrm{s}$, and bearing in mind that there is an improved synergy with current digital video broadcast concepts (e.g. MPEG) when using a more advanced algorithm, the choice for 16:8:0 sampling can be well substantiated, since it yields:

- the best extensions of digital SD formats;

- consistency between the 50 - and $60 \mathrm{~Hz}$ world;

- sufficient quality gain as compared to SDTV;

- synergy with digital transmission concepts.

\subsection{Compression algorithm}

The desired output rate of $50 \mathrm{Mbit} / \mathrm{s}$ implies a compression factor 10 , which can be obtained by a DCT-based coding scheme using interframe coding. Interframe coding using motioncompensated prediction has been proven to be very efficient for transmission applications. However, the special requirements of tape recording restrict the extensive use of interframe coding. Under such conditions, it is a good compromise to apply intra-two-frame coding with predictive coding of every second frame. In addition, the system description shows that our scheme can be extended to an MPEG architecure, thereby enabling flexibility for the future.

Figure 2 portrays an example of a scheme in which only every odd frame is DCT coded directly, while the even frames are skipped and reconstructed by motion-compensated prediction 


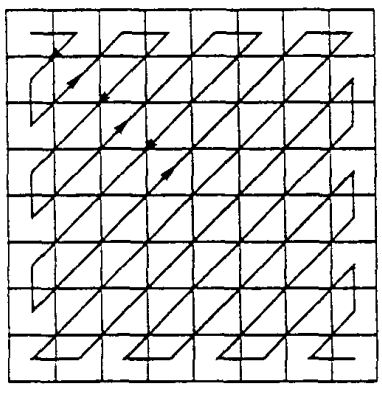

(a) Static mode.

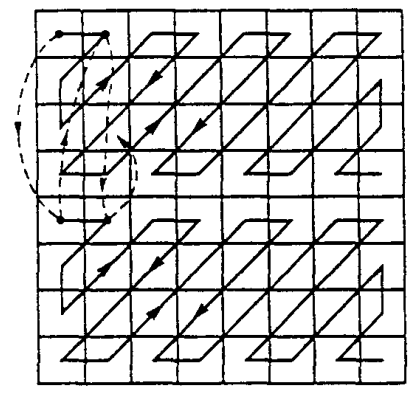

(b) Motion mode.

\begin{tabular}{l|rrrrrrrrr} 
& 0 & 1 & 2 & 3 & 4 & 5 & 6 & 7 & 8 \\
\hline 0 & 11 & 2 & 3 & 4 & 4 & 4 & 5 & 6 & 6 \\
1 & 11 & 4 & 5 & 7 & 7 & 8 & 9 & 9 & 10 \\
2 & 12 & 5 & 7 & 9 & 10 & 11 & 11 & 12 & 13 \\
3 & 13 & 5 & 8 & 10 & 11 & 12 & 13 & & \\
4 & 13 & 6 & 9 & 11 & 13 & 13 & & & \\
5 & 13 & 6 & 10 & 12 & & & & & \\
6 & 13 & 7 & 10 & 12 & & & & & \\
7 & 13 & 7 & 11 & 13 & & & & & \\
8 & 13 & 7 & 11 & & & & & & \\
9 & 13 & 8 & 12 & & & & & & \\
10 & 13 & 8 & 13 & & & & & & \\
11 & 13 & 8 & & & & & & \\
12 & 14 & 8 & & & & & &
\end{tabular}

Table 3: Codeword lengths.

Figure 5: Coefficient scanning patterns in different modes (left and middle) and VLC encoding table (right), showing the word lengths (without sign bit) as a function of the runlength (vertical direction) and the amplitude (horizontal direction).

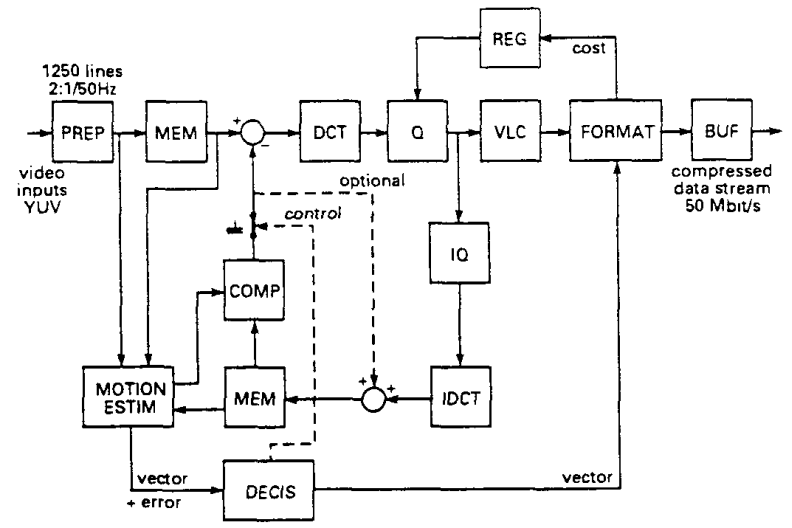

Figure 2: Encoder block diagram of the HDTV video compression system.

(COMP), using the adjacent frames and the transmitted prediction error. If the switch is in the open position, there is no subtraction and the incoming frame is coded directly with intraframe DCT coding techniques (DCT $+Q)$. Simultaneously at the output, the compressed video is reconstructed by inverse quantization and tranformation (DCT $+\mathrm{Q}^{-1}$ ) and stored in the memory (MEM) of the feedback prediction loop. At the start of the second frame, the switch is closed and the stored reconstructed frame is used to per- form first motion-estimation (MOTION ESTIM) to calculate the motion vectors, and secondly, apply motion-compensated prediction (COMP) for the new (even) frame. The difference between the prediction and the second frame is then coded, using the same DCT coding scheme. As the compensated prediction is sometimes not reliable due to complex motion (e.g. in the case of scene cuts), the quality of the prediction is analysed (DECIS) to switch to unpredicted (fallback) coding, if required. This decision can be made on block basis. Let us now consider some details of the bit-rate reduction algorithm.

Basically, two prediction schemes can be implemented, namely, either one-sided prediction (previous frame) or two-sided prediction (surrounding frames, see Fig. 3). In both approaches, the motion in frames is estimated by measuring the displacements - denoted by $\vec{D}$ - of objects, so that a good prediction and thus a high coding efficiency is obtained. We have described in [7] that two-sided prediction is more efficient, even if the accuracy is increased to subpixel accuracy in the one-directional case. Considering the importance of a good picture quality for HDTV applications, the two-sided prediction has been implemented, although it requires more processing hardware and larger memory.

The $8 \times 8$ sample blocks for DCT coding are constructed within a frame to improve the cod- 


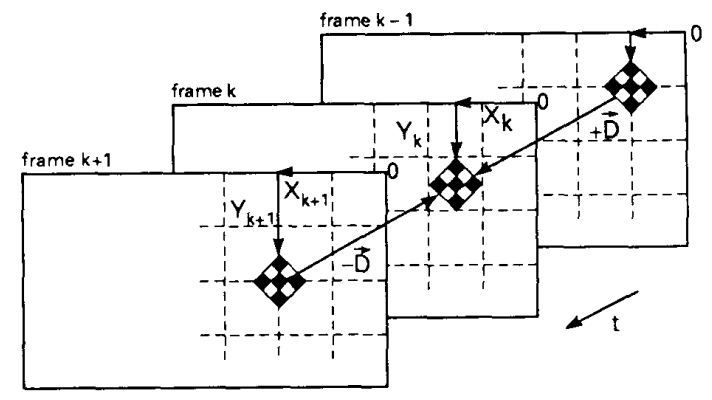

Figure 3: Principle of two-sided motioncompensation, using the surrounding frames.

ing efficiency. At moving edges, however, framebased blocks contain considerable energy on high vertical frequencies. This penalty can be overcome by using a motion detector and subsequently performing an $8 \times 8 \mathrm{DCT}$ in static areas, whereas in the case of motion, a $4 \times 8$ field transformation is carried out twice (see [8]). The signal components are merged for subsequent quantization and coding.

The spectral components, i.e. DCT coefficients, are quantized using a 'frequencydependent' weighting and adaptive uniform quantization. More coarse quantization of the high-frequency components (human eye characteristics) can be accomplished by using a multiplicative weighting factor for each individual coefficient (see e.g. [2]). Additionally, adaptive quantization is employed to exploit the difference in perception between quantization errors in flat areas and structured regions. This adaptivity (4 classes) is implemented by a variable quantizer step size which is controlled by the 'activity' of a block, e.g. the maximum ac coefficient of each individual DCT block. Finally, the quantizer is also controlled by the buffer status (block REG in Figure 2) to obtain a fixed bit cost for every set of two frames (editing).

For further compression of the quantized coefficients, the well-known zig-zag scanning and variable-length coding are performed. As indicated in Figure 5, the scanning of coefficients is different for static $(8 \times 8)$ and 'motion' (two times $4 \times 8$ ) blocks.
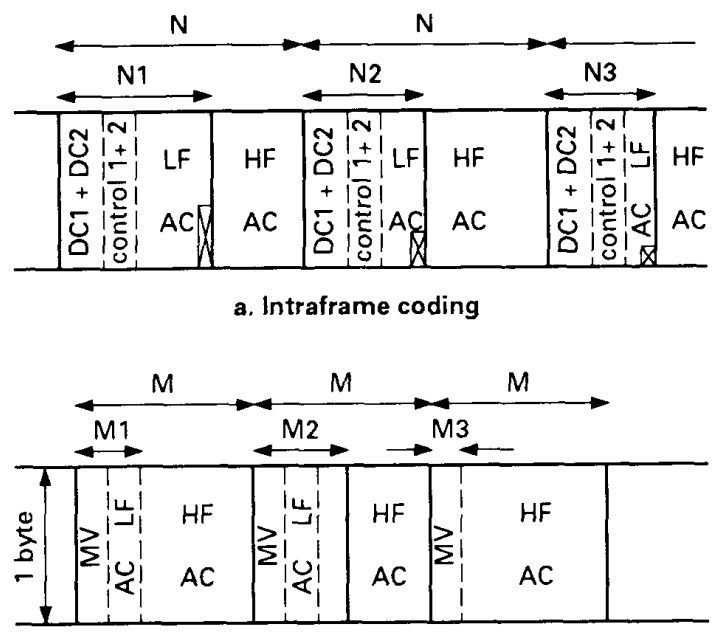

b. Interframe coding

Figure 4: VLC output format using a separation of most important and less relevant data. (DC = dc coefficient, $\mathrm{AC}=\mathrm{ac}$ coefficient, $\mathrm{MV}=$ motion vector data, $L F=$ low-frequency coefficients.

The variable-length coding technique is based on zero runlength coding, as considered in the MPEG algorithrn. The length of a string of consecutive zero coefficients (the runlength) is counted and is combined with the subsequent non-zero coefficient into one codeword of variable length (see Table 3). Each block is terminated with an EOB codeword. The same technique is applied to coding of the motion-compensated frame.

To increase the error robustness and to enhance VLC signal recovery during the trick modes, a 'self-synchronizing' code (see e.g. [9]) is applied. Taking into account the required throughput rates, the codeword length has been limited to 16 bits.

\subsection{Robust output format}

When the full capacity of the error-correction system is not available, e.g. during very large burst errors exceeding the correcting capabilities in normal playback or during the trick modes, a robust video format is particularly useful.

The basic features of the VLC output format are best explained by Figure 4. A key aspect of 
this format is to separate most important data from less relevant data for pictorial reconstruction. In our case, most important data is always low-frequency (LF) coefficient data, including the dc coefficient (DC) and quantizer control bits in intraframe mode (first frame), and motion vectors in the interframe mode (second frame). Less relevant data contains the remaining highfrequency (HF) coefficient data.

The separation is performed as follows. From every group of DCT blocks we extract the LF $\mathrm{AC}$ data as an integer number of bytes, e.g. $N 1$ bytes for the first group in the picture. This LF data is then put on fixed positions in the output bit stream. In Figure 4, these positions are at the start of every $N$ bytes in intraframe mode and every $M$ bytes in the interframe mode. The amount of LF bytes is variable, but is uniquely defined by the transmitted quantizer control bits, so that no extra overhead is required.

The fixed positions of the most relevant data enable a high robustness, since this data can always be found, regardless of possible error propagation of the variable-length decoder. Moreover, during trick-mode replay, the LF AC data portions can be used for reconstructing a complete 'low-frequency' image, without the need to resynchronize on the HF AC data stream for variablelength decoding. Basically, a simple byte counter is sufficient to locate the LF AC data. In our implementation, we used two DCT blocks in a group for simplicity, but this format can be extended easily to macroblock basis (6 DCT blocks).

\section{Channel coding}

\subsection{Error-correcting code}

The task of the error-correcting code is to protect the compressed digital video signal and the audio signal against random errors, due to the noisy channel, and burst errors resulting from dropouts, scratches, etc. For this purpose, the wellknown Reed-Solomon product code is adopted, consisting of an inner and an outer code [10] [5]. The structure of the data in a track is visualized in Figure 6.

The track is divided into two track parts, each consisting of a video and an audio ErrorCorrecting Code (ECC) block. The format with the gaps allows for separate editing of the video

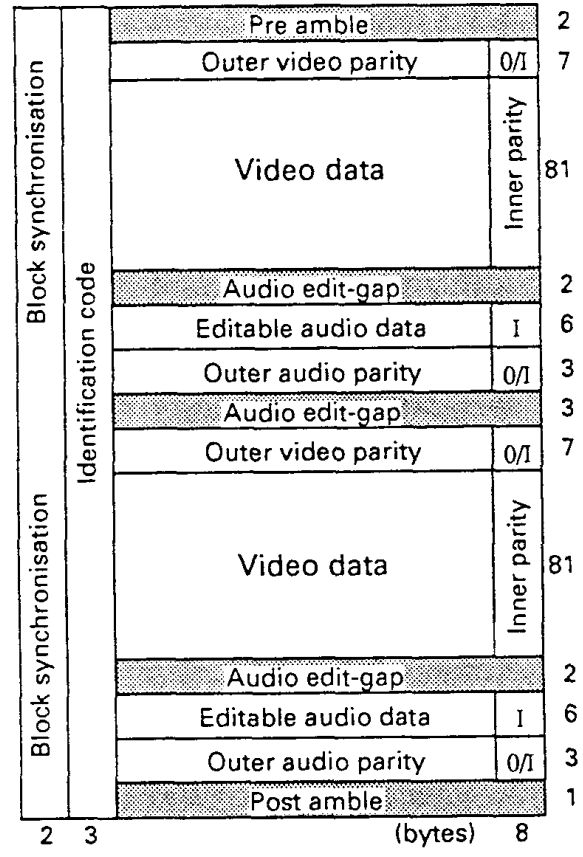

Figure 6: Track format of the system, showing the use of product codes for video and audio.

and the audio data. Moreover, it offers the flexibility to use smaller scanners in the future, as the track format can be split into two parts.

The inner ECC, requiring a redundancy of 6 $\%$, is identical for the video and audio signals, and its main task is protection against random errors. The outer code is particularly useful for the correction of burst errors, due to scratches, and requires a redundancy of $8 \%$ for the video part. The outer code for the audio part requires a redundancy of $33 \%$.

The error protection should not only supply error-free data in normal playback mode, but also deliver as many error-free video blocks as possible in trick modes (i.e. playback speeds that differ from the nominal value). For the trick modes, we distinguish picture search and slow motion. In picture search, the outer ECC cannot be applied, because not all of the inner code blocks related to one product ECC block are received.

In slow motion, however, all data from one product block can be gathered, but the data is not retrieved in a sequential order. This is due 
to the multiple scanning of all track parts for such low speeds. As a result, the outer ECC can be activated during slow motion. This is important, because displayed pictures are repeated on the screen, so that errors become more visible.

The performance in trick modes can be investigated and the maximum tracking error for which the quality of the data detection is within the regular boundaries can be determined. An error statistics measurement and evaluation option has been incorporated. One of the results that we have found is that head clogging is a very important source of errors, especially because it seems to be initiated by drop-outs on the tape. This type of error normally leads to burst errors ranging from e.g. $10 \%$ of the track to a few tracks. The kind of head clogging discussed here is self cleaning, but occurs much more often than was expected.

\subsection{Digital audio}

As is visualized in Figure 6, two separate audio regions are available in the track format, surrounded by edit gaps. This allows for individual editing of the audio information. Each region has sufficient capacity to accommodate a complete stereo channel, having a sampling frequency of $48 \mathrm{kHz}$ and an accuracy of 16 bits. Since they are stored in different sets of 6 tracks, the left and right channel are recorded separately on the tape, thereby allowing for editing on a mono channel basis. Shuffling of the audio data is applied to enhance the error concealment performance. In principle, other sampling standards, such as 44.1 $\mathrm{kHz} / 16$ bits linear or $32 \mathrm{kHz} / 12$ bits non-linear, can also be recorded. In the latter case, 8 individual audio mono channels are available to the user. This is important for HDTV broadcasts or prerecorded software with multiple audio channels (multi-lingual). The $48 \mathrm{kHz} / 16$ bits mode is incorporated in the demonstration model (based on programmable DSPs).

\subsection{4-25 modulation}

For optimizing the recording channel, we use 2425 modulation with partial-response detection. The purpose of channel modulation is spectral shaping in order to adapt the bit stream to the characteristics of the recording channel. This matching refers not only to the detection method, but also to other demands, such as the possibility of Automatic or Dynamic Track Following (ATF and DTF).

For improved bit detection, the modulation imposes constraints on the runlength of bit sequences of equal polarity and it reduces the DC energy of the recording signal. For ATF and DTF, we designed a 24-25 modulation technique with embedded tracking tones, because this is well suited for digital recording [2].

Using the 24-25 modulation, the spectrum of the recording bit stream is shaped such that adequate pilot frequencies are present. During playback, the crosstalk from the pilots in the neighbouring tracks is used as tracking information. The power of the spectral components on and around the pilot frequencies used for both the in-track code signal and the crosstalk from the neighbouring tracks should be minimized for improved tracking detection.

In the playback channel, the well-known Partial-Response Class IV (PR4) equalisation (see [11]) with Viterbi detection is used [12] [13] [14]. In order to prevent error propagation at playback, a technique known as Interleaved NRZI modulation is applied. This is realized by the insertion of a $2 \mathrm{~T}$ precoder in the record path. As a consequence, a single bit inserted in the total bit stream before precoding, influences the polarity of all the subsequent bits in one of the two interleaved recording bit streams, so that the DC energy can be controlled. If these extra bits are inserted regularly, the spectrum of the recording signal can be adjusted such that it is DC free, contains spectral notches and has pilot tones. In addition, the extra bits from the 24-25 modulation can be used for run-length limiting, due to the interleaved nature of the modulation technique, offering the opportunity to use an almost unique synchronization word.

Because of the byte-based structure of the surrounding error-correcting code, it is advantageous to insert the extra bit between an integer number of bytes, leading to coding schemes like 8$9,16-17,24-25,32-33$, etc.. It is important to notice that, with these schemes, the extra code bit occurs alternatingly in both interleaved streams, so that the polarity of all the bits in the recording bit stream can be influenced. 


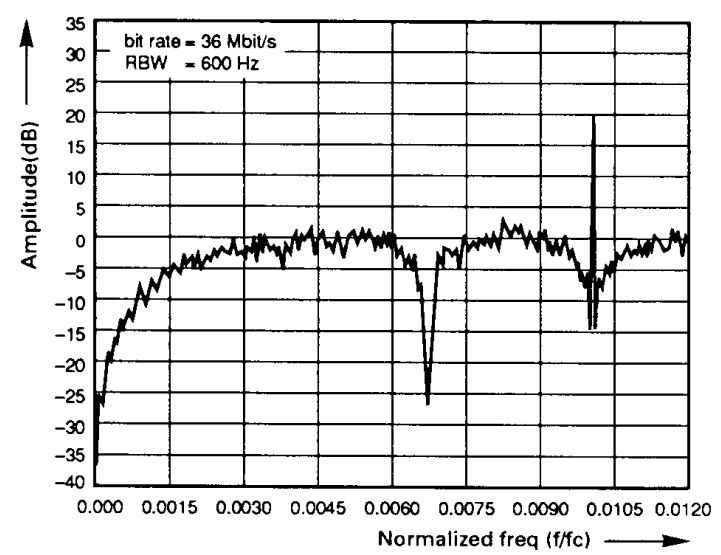

Figure 7: Code spectrum containing DC-free area, notch and pilot frequency (left to right).

It will be clear that coding tables are not required for the aforementioned mapping. For the choice of the polarity of the extra bit, a 'spectrum analyser' is connected to the output of the $2 \mathrm{~T}$ precoded bit stream. In this analyser, the desired pilot tone is first subtracted from the signal, and then the spectral power in the recording bit stream at DC and around the pilot frequencies is determined. The extra bit is chosen such that these powers are minimized, resulting in a DC-free spectrum with notches at the pilot frequencies, and a pilot at the desired frequency.

If no pilot is subtracted, a similar spectrum without pilot is obtained. The verification of the runlength constraint (in our implementation maximal 9) is performed separately and it overrules the polarity choice of the analyser, if required.

In the real-time prototype, we opted for three different spectra, indicated by $f_{0}, f_{1}$ and $f_{2}$, which are applied in subsequent tracks using the order $f_{0}, f_{1}, f_{0}, f_{2}, f_{0}, f_{1}$, etc.. A spectrum without pilot is indicated by $f_{0}$. The spectra $f_{1}$ and $f_{2}$ contain a pilot at $f_{c} / 150$ and $f_{c} / 100$, respectively, where $f_{c}$ stands for the bit clock in the recording bit stream. The spectrum $f_{2}$ is given in Figure 7. Because of the adopted two-channel recording system, the pilot $f_{0}$ is recorded in channel 1 and the pilots $f_{1}, f_{2}$ are recorded in channel 2. During playback, only channel 1 is used for

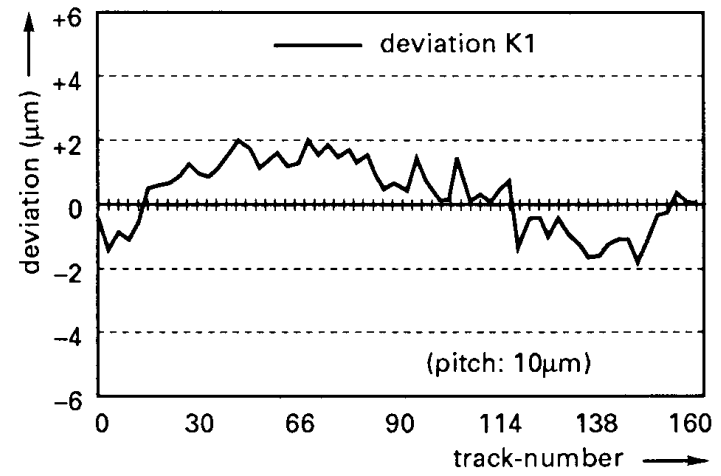

Figure 8: Measurement of track linearity.

the detection of the tracking signal. Because the crosstalk of both pilots is read by the same head, a high robustness against gain variations is obtained.

Decoding of the 24-25 modulation is very simple, since the extra bits only have to be discarded from the detected bit stream. The position of these bits is easily derived from the fixed sync block length, which is a multiple of three bytes ( 25 bits in the channel). Hence, the extra bits occur in a fixed position in the sync block.

\section{Mechanical deck and scanner}

The recording system is based on $8 \mathrm{~mm}$ mechanics with a $40 \mathrm{~mm}$ scanner. The analogue video- 8 system has an effective track length of roughly 62 $\mathrm{mm}$ using $180^{\circ}$ wrap angle and a track width of $34 \mu \mathrm{m}$ ( $50 \mathrm{~Hz}$ environment). To improve the (digital) recording density, the recorded track width is reduced to $10 \mu \mathrm{m}$, using guardbandless azimuth recording.

The relatively high recording rate of $72 \mathrm{Mbit} / \mathrm{s}$ is processed by a two-channel system, resulting in a scanner with four record-playback heads. Metal-evaporated (ME) tapes with newly developed TSS heads enable a high linear recording density.

\subsection{Mechanical deck}

The aforementioned density requires an improved mechanical stability and accuracy, so that the deck was modified to obtain sufficient stiff- 
ness and robustness. Head window and scanner surface were modified to improve the head-tape contact at the increased scanner speed of 4500 rpm. After carefully checking the tape path and the tape tension, we achieved a typical track linearity as shown in Figure 8.

Writing two $10 \mu \mathrm{m}$ tracks in parallel without guardband, results in a tape velocity of 3.5 $\mathrm{cm} / \mathrm{s}$. The nominal playing time for a $90-\mathrm{min}$. ME analogue video- 8 cassette is roughly $60 \mathrm{~min}-$ utes. However, as the trend in tape technology is towards thinner tapes, the playing time can gradually increase to 2 hours.

The deck and servo system support a picture search mode and slow motion. By using a programmable system for servo functions (see Section 5.3), the tape speed can be controlled with high accuracy and flexibility. For preliminary experiments, a search mode of 3-3.5 times the normal tape speed has been implemented in both directions, while slow motion is possible at $1 / 3$ of the normal tape speed, both in forward and backward mode.

Although longitudinal tracks can be omitted, due to the ATF information (pilot tones) in the helical tracks, a control track at the bottom tape edge was implemented for experimental purposes at the initial stage when the tones were absent. The ergonomical system design enables the user to control the tape recorder in a convenient way by means of an LCD-display and soft keys.

\subsection{Scanner}

The scanner assembly consists of the stationary lower drum with a small sensorless and brushless motor and the rotating upper drum. Highly reliable ball bearings enable stable rotation of the upper drum. Between the two drum parts, a two-channel rotary wide-band transformer is placed for data transfer. The active playback circuitry is located inside a shielded cylindrical part of the rotating upper drum. The angle between the heads of the same head pair is $8^{\circ}$. Figure 9 portrays the construction of the scanner system.

The preamplifier circuitry has been mounted in the rotating upper drum to realize short connections between heads and preamplifier. A second advantage is to have no transformer leakage inductance and stray capacitance on the input of

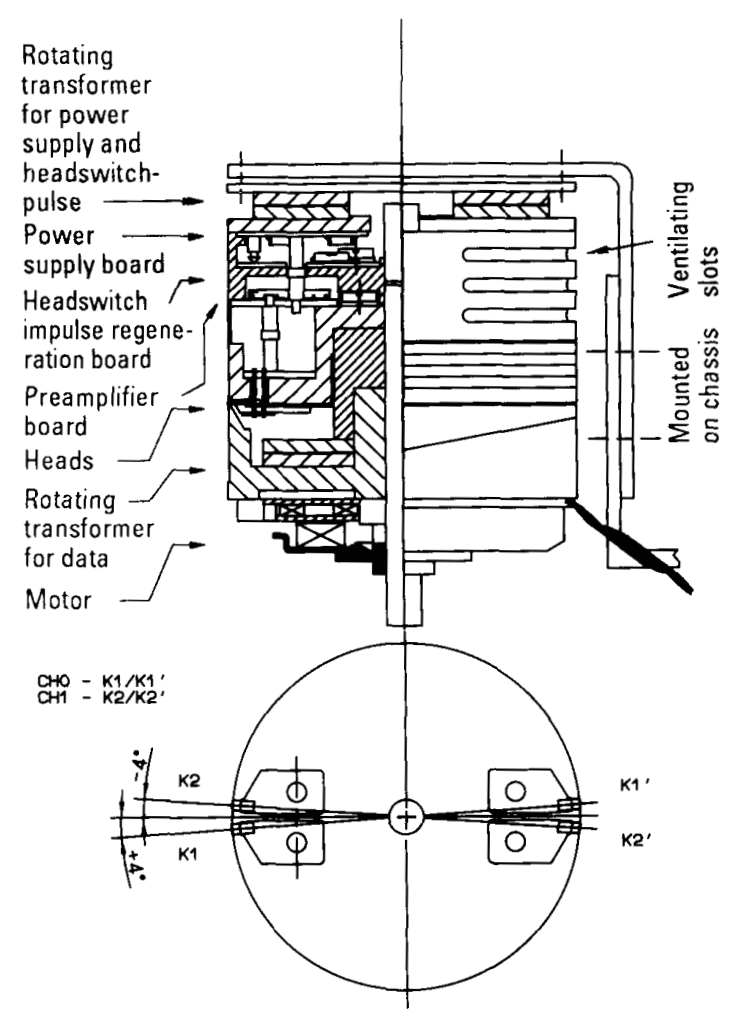

Figure 9: Construction diagram of scanner with rotating preamplifier.

the amplifier. A third benefit, resulting from the shielded and grounded drum, is the protection of the amplifier against RF-interference from outside.

The amplifier has been implemented with SMD technology. The record/playback-switching circuitry is based on a small special relay device. The relays are mounted on the circuit board in such a way that the centrifugal forces from the rotating drum do not influence the relaycontact function. Playback head-switching circuitry on the drum makes it possible to use only one two-channel transformer for 4 video heads. The power supply and head-switch pulse transfer are carried out by an extra transformer on top of the scanner. The power supply is activated during the playback mode. The block diagram of the active playback circuitry is shown in Figure 10 . 


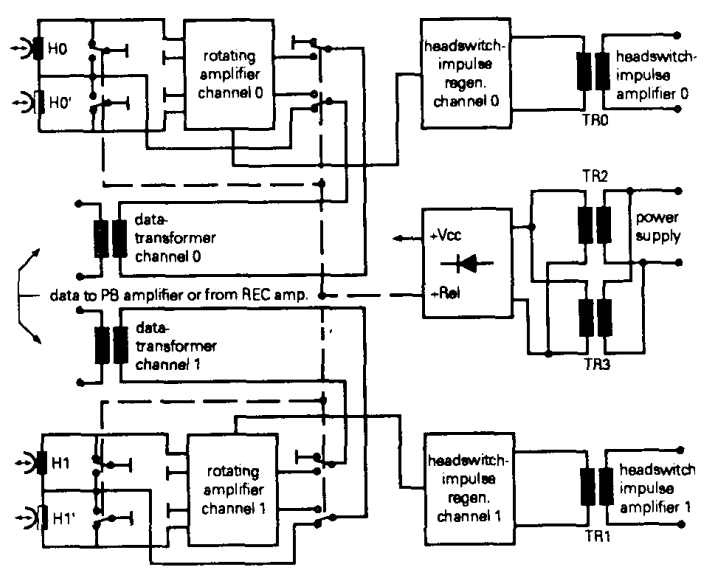

Figure 10: Block diagram of scanner system.

\subsection{Servo system}

The servo system has been designed around a high-speed microcomputer (see Fig. 11), which controls all main mechadeck functions, communication, keyboard, display, and it performs the regulation control for the scanner and tape transport systems. The software-based design is perfectly suited for the fast transfer of parameters between related regulation systems; this is especially required in the implemented trick modes, ranging from slow motion up to 8 times search speed in forward and backward direction.

A new algorithm for the dynamic compensation of mechanical and magnetical tolerances inside the scanner speed feedback generator reduces the total scanner jitter with the tape in contact within a margin of $3 \mu s$. The scanner phase control is active on a video frame basis, so that the recorder quickly responds to video source switching. The tape transport system is equipped with a closed frequency-loop system and an ATF-phase control in playback mode, which guarantees fast tracking lock conditions, combined with the lowest possible tracking errors. Although the tape transport system is designed to lock on one particular ATF pattern out of six equal patterns resulting from the multitrack format (24 tracks / 2 frames), the system locks on the first valid ATF block to improve synchronization response time. The variable starting point for video decoding is compensated by the

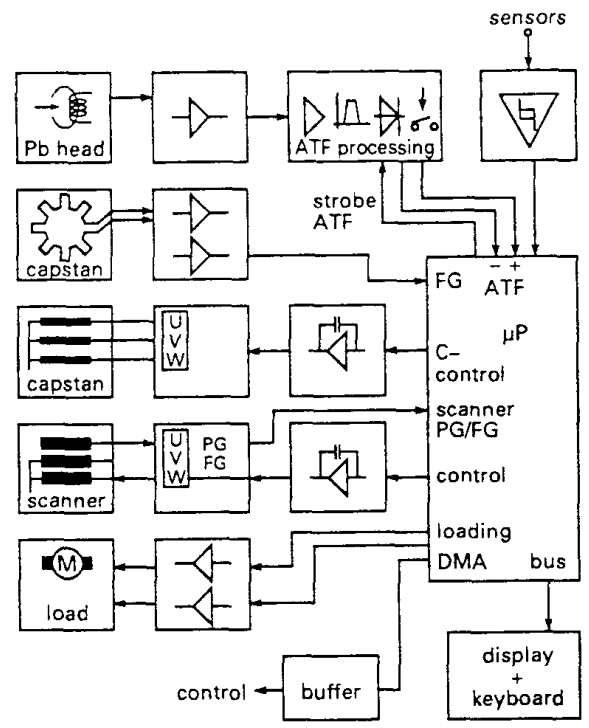

Figure 11: Architecture of servo system.

use of a large buffer memory.

An RS-232 bus supports communication between the servo system and external electronics, and allows to check and analyze various parameters, such as the deck status, tape counter, or equalizing setting. The system incorporates a monochrome LCD-display with $240 \times 60$ dots to monitor status, user commands, icons, and on request, 'hidden' parameters, like head switching delay and motor parameters for technical services.

\section{Conclusions}

The concept and real-time implementation of an experimental home-use digital HDTV recorder has been presented. The system is based on small (modified $8 \mathrm{~mm}$ ) recording mechanics and cassette and it uses advanced bit-rate reduction. The bit rate is sufficiently low (50 Mbit/s) for a cost-effective implementation of both HDTV and SDTV recording on the same mechanics.

The bit-rate reduction algorithm is based on advanced motion-compensated DCT coding and is organized in such a way that it can be extended to MPEG applications, although a number of measures have been included to support and enhance editing (fixed compression in two 
frames) and trick-mode replay (special output formatting).

The error-correcting code is based on two product code blocks on one track to have flexibility for other future tape formats. The newly developed 24-25 channel code, featuring digitally embedded pilot tones for tracking, has a high efficiency (only $4 \%$ overhead) and low complexity (no tables), while it enables sufficient monitoring capability of the tracking tones during playback (and DTF possibility).

The scanner was optimized for the high channel rates, using $2 \times 2$ heads, and a rotating preamplifier has contributed to an improved signal-tonoise ratio at the bit-detection side during playback. A software-based servo design allows for high flexibility and improved accuracy in the recording system control.

\section{Acknowledgements}

A large note of appreciation goes to Ing. Joost A.H.M. Kahlman (Philips Research), who contributed significantly to the design of the 2425 channel code. We also greatfully acknowledge many colleagues of Philips Research Labs., Thomson Consumer Electronics Labs. and Grundig Research and Predevelopment Labs. Special thanks to Ir. R. Brondijk and Ing. F. Brüls (ASICs), Ir. A. Denissen, Ing. J. Kahlman, Dipl.-ing. K. Knuth, Dipl.-ing. A. Kluger, Ing. M. Looykens, and MSc. C. Wong (channel electronics), Dipl.-ing. M. Drexler, Ing. O. Eerenberg, Ing. E. Jaspers, Ing. F. Jorritsma, and Dipl.-ing. R. Schäfer, (video coding), Dipl.-ing. W. Bachnik and Dipl.-ing. W. Vössing (audio), Mr. Dietz, Mr. H. Liebmann, Mr. P. Mahr, and Mr. R. Zöllner (mechanics and control).

\section{References}

[1] P.H.N. de With, M. Breeuwer, P.A.M. van Grinsven, 'Data Compression Systems for Home-Use Digital Video Recording', IEEE Journ. Sel. Areas Commun., Vol. 10, No. 1, pp. 97-121, January 1992.

[2] S.M.C. Borgers et al., 'An Experimental Digital VCR with 40-mm Drum and DCTBased Bit-Rate Reduction', IEEE Trans. Consum. Electron., Vol. CE-34, No. 3, pp. 597-605, August 1988.
[3] M. Yoneda et al., 'An Experimental Digital VCR with New DCT-Based Bit-Rate Reduction System', IEEE Trans. Consum. Electron., Vol. CE-37, No. 3, pp. 275-282, August 1991.

[4] N. Doi et al., 'Adaptive DCT coding for home digital VTR', IEEE Proc. Global Telecomm. Conf., Hollywood (USA), Vol. 2, pp. 1073-1079, November 1988.

[5] C. Yamamitsu et al., 'An Experimental Study for Home-Use Digital VTR', IEEE Trans. Consum. Electron., Vol. CE-35, No. 3, pp. 450-457, August 1989.

[6] K. Onishi et al., 'An Experimental HomeUse Digital VCR with Three-Dimensional DCT and Superimposed Error Correction Coding', IEEE Trans. Consum. Electron., Vol. CE-37, No. 3, pp. 252-260, August 1991.

[7] P.H.N. de With et al., 'Digital Consumer HDTV Recording Based on MotionCompensated DCT Coding of Video Signals', Sign. Proc. Image Commun., Vol. 4 , Nos. 4-5, pp 401-420, August 1992.

[8] P.H.N. de With, 'Motion-Adaptive Transform Coding of Video Signals', Philips Journ. Res., Vol. 44, Nos. 2/3, pp. 345-364, 1989.

[9] B.L. Montgomery and J. Abrahams, 'Synchronization of Binary Source Codes', IEEE Trans. Inform. Theory, Vol. IT-32, No. 6, pp. 849-854, November 1986.

[10] S. Gregory, 'Introduction to the 4:2:2 Digital Video Tape Recorder', Pentech Press, London, 1988, Chapter 6 and Appendix A.

[11] K.A.S. Immink, Coding Techniques for Digital Recorders, Prentice-Hall, Inc., Englewood Cliffs, New Jersey, 1990.

12] L.M.H.E. Driessen et al., 'An experimental digital video recording system', IEEE Trans. Consum. Electron., Vol. CE-32, No.3, pp. 362-371, August 1986.

[13] C. Yamamitsu et al., 'An Experimental Study on Bit-Rate Reduction and HighDensity Recording for Home-Use Digital VTR', IEEE Trans. Consum. Electron., Vol. CE-34, No. 3, pp. 588-596, August 1988.

[14] R.J. van der Vleuten and K.A.S. Immink, 'A Maximum Likelihood Detector for a Class IV Partial-Response Magnetic Recording System', Proc. 10th Inform. Theory Sympos. Benelux, Houthalen, Belgium, pp. 117123, May 1989. 